A method of structural and parametric assessment of the object state has been developed. The essence of the method is to provide an analysis of the current state of the object under analysis. The key difference of the developed method is the use of advanced procedures for processing undefined initial data, selection, crossover, mutation, formation of the initial population, advanced procedure for training artificial neural networks and rounding coordinates. The use of the method of structural-parametric assessment of the object state allows increasing the efficiency of object state assessment. An objective and complete analysis is achieved using an advanced algorithm of evolution strategies. The essence of the training procedure is the training of synaptic weights of the artificial neural network, the type and parameters of the membership function, the architecture of individual elements and the architecture of the artificial neural network as a whole. An example of using the proposed method in assessing the operational situation of the troops (forces) grouping is given. The developed method is 30-35\% more efficient in terms of the fitness of the obtained solution compared to the conventional algorithm of evolution strategies. Also, the proposed method is 20-25\% better than the modified algorithms of evolution strategies due to the use of additional improved procedures according to the criterion of fitness of the obtained solution. The proposed method can be used in decision support systems of automated control systems (artillery units, special-purpose geographic information systems). It can also be used in DSS for aviation and air defense ACS, DSS for logistics ACS of the Armed Forces of Ukraine

Keywords: artificial neural networks, neural network training, modified algorithm of evolution strategies

\section{DEVELOPMENT OF A METHOD OF STRUCTURAL-PARAMETRIC ASSESSMENT OF THE OBJECT STATE}

Qasim Abbood Mahdi $\mathrm{PhD}$, Head of Department Computer Technologies Engineering Department Al Taff University College Karrada str., 3, Karbala, Iraq, 31001 Rus Ian Zhyvotovskyi Corresponding author

$\mathrm{PhD}$, Senior Researcher, Head of Research Department Research Department of Development Armament and Military Equipment of Air Force* E-mail: ruslan_zvivotov@ukr.net Serhi i Kravchenko $\mathrm{PhD}$, Associate Professor Department of Software Engineering National Aviation University Liubomyra Huzara ave., 1, Kyiv, Ukraine, 03058

I hor Borysov

$\mathrm{PhD}$, Associate Professor, Head of Research Department Research Department of Problems of Research of Means of Communication and Automation Military Unit A1906

Melnikova str., 81, Kyiv, Ukraine, 04050

O leks andr Orlov Doctor of Sciences in Public Administration, Professor, Head of Department Department of Digital Technologies and Electronic Government Research and Educational Institute of Public Administration V. N. Karazin Kharkiv National University Svobody sq., 4, Kharkiv, Ukraine, 61022

Ihor Panchenko $\mathrm{PhD}$, Head of Department Department of Special Information Systems and Robotic Complexes $* * *$

Yevhen Zhyvylo $\mathrm{PhD}$, Head of Department Department of Military Training***

Artem Kupch y Military-Technical Policy Department*

D m y tro Koltovskov Adjunct**

Serhii Boholii Adjunct**

*Central Scientific Research Institute of Armament and Military Equipment of the Armed Forces of Ukraine Povitrofloski ave., 28, Kyiv, Ukraine, 03049

**Scientific and Organizational Department***

$* * *$ Military Institute of Telecommunications and Information Technologies named after Heroes of Kruty Moskovska str., 45/1, Kyiv, Ukraine, 01011
Received date 02.08.2021 Accepted date 13.09.2021 Published date 29.10.2021
How to Cite: Mahdi, Q. A., Zhyvotovskyi, R., Kravchenko, S., Borysov, I., Oleksandr, O., Panchenko, I., Zhyvylo, Y., Kupchyn, A., Koltovskov, D., Boholii, S. (2021). Development of a method of structural-parametric assessment of the object state. EasternEuropean Journal of Enterprise Technologies, 5 (4 (113)), 34-44. doi: https://doi.org/10.15587/1729-4061.2021.240178

\section{Introduction}

Decision support systems (DSS) are actively used in all spheres of human life. They are especially common in the processing of large data sets in databases, process development forecasting, providing informational and analytical support to the decision-making process of decision-makers. 
Existing DSS are based on statistical and artificial intelligence methods, which provide collection, processing, generalization of information about the state of objects (processes) and forecasting their future state.

The creation of intelligent DSS has become a natural continuation of the widespread use of conventional DSS. The main fundamental difference between intelligent DSS and conventional DSS is the presence of feedback and adaptability to changes in input processes [1,2].

Intelligent DSS have been widely used to solve specific military tasks, namely [1,2]:

- planning the deployment, operation of communication and data transmission systems;

- automation of troops and weapons control;

- collection, processing and generalization of intelligence information about the state of intelligence objects, etc.

The structure of intelligent DSS can be conventionally divided into 4 major layers:

- interface layer (interactivity and visualization);

- modeling layer (statistical models and machine learning; numerical models; game theory models, probability-statistical methods, etc.);

- data processing layer (data flow organization, work with databases and expert assessments);

- data collection layer (web scanning, sensors and programming interface).

Analysis of the experience of creating intelligent DSS shows that the most promising for their construction is an information technology based on a combination of different approaches [2-5]. One such approach is a combination of evolution strategies and artificial neural networks (ANN). All this allows processing different types of data, adapting the structure to the type and amount of input data, thereby increasing performance.

Evolutionary methods compared to conventional approaches have the following advantages [6-8]:

- the ability to quickly adapt to the subject area, which almost without any changes allows forming a population and ANN structure that corresponds to this process;

- the ability to conduct a parallel solution search in several directions;

- avoiding the problem of falling into the trap of the local optimum;

- the ability to work under a priori uncertainty, nonlinearity, stochasticity and chaos, all kinds of disturbances and interference;

- have universal approximating capabilities.

Evolutionary methods are widely used to solve various problems of data mining, planning, control, identification, emulation, forecasting, intelligent control, etc. on each layer of intelligent DSS. Despite their successful application to a wide range of data mining problems, these systems have a number of disadvantages. The most significant shortcomings are as follows:

1. The complexity of choosing the system architecture. As a rule, the model based on the principles of computational intelligence has a fixed architecture. Therefore, adapting the system to new data that are different from previous data may be problematic.

2. Training in batch mode and training for several epochs require significant time resources. Such systems are not adapted to work online with a fairly high rate of new data.

3. Problems in taking into account indicators that have a complex structure of relationships and contradict each other.

4. The difficulty of considering the indirect influence of interdependent components under uncertainty.
Thus, the combination of the method of evolution strategies and ANN allows analyzing (assessing) the state of analysis objects and conducting deep learning of databases. This, in turn, determines the relevance of research to improve the efficiency of structural and parametric assessment of monitoring objects.

\section{Literature review and problem statement}

The work [9] presents a hybrid evolution strategy that helps architects generate sets of floor plans at an early design stage. This approach is based on the algorithm of evolution strategies. The algorithm is an advanced evolution strategy with a stochastic hill climbing technique. The disadvantages of this approach include low efficiency, lack of consideration of the type of uncertainty about the analysis object state and accumulation of evaluation errors. The accumulation of evaluation errors is caused by the imperfection of the learning algorithm.

The work [10] proposes a dynamic multi-purpose evolutionary algorithm for processing different data. The disadvantages of this approach include low efficiency, lack of consideration of the type of uncertainty about the object state and lack of approaches to learning this algorithm.

The work [11] presents the use of machine learning methods, namely ANN and genetic algorithms. A genetic algorithm is used as a method of ANN training. The disadvantages of this approach are limited training of only synaptic weights, without training the type and parameters of the membership function. This, in turn, causes the accumulation of learning errors.

The work [12] presents the use of machine learning methods, namely ANN and differential search method. In the course of the research, a hybrid method of ANN training was developed, based on the backpropagation algorithm and differential search. The disadvantages of this approach are limited training of only synaptic weights, without training the type and parameters of the membership function.

The work [13] developed ANN training methods using the combined approximation of the response surface, which provides the smallest training and forecasting errors. The disadvantage of this method is the accumulation of training errors and the inability to change the ANN architecture during training.

The work [14] shows the use of ANN to evaluate the efficiency of the unit, using the previous time series of its performance. SBM (Stochastic Block Model) and DEA (Data Envelopment Analysis) models are used for ANN training. The disadvantages of this approach are the limited choice of network architecture and training only synaptic weights.

The work [15] shows the use of ANN for estimating traffic intensity. The backpropagation algorithm is used as a method of ANN training. The performance of the backpropagation algorithm can be improved by using bandwidth connections between each layer, so that each layer sets out only the residual function relative to the results of the previous layer. The disadvantage of this approach is limited training of only synaptic weights without training the type and parameters of the membership function.

The work [16] shows the approach of estimating input data for decision support systems. The essence of the proposed approach is to cluster the basic set of input data, analyze them, and train the system based on the analysis. The disadvantages of this approach are the gradual accumulation of estimation errors, low efficiency and lack of consideration of a priori uncertainty about the object state. 
The work [17] considers the method of evolution strategies for improving the evolutionary ability of robot swarms. The method uses the swarm coevolution mechanism to increase the evolution efficiency. A behavior expression tree is used, which expands the search space for the developed strategies. The results demonstrate the advantage of the proposed one over the conventional algorithm of evolution strategies in terms of evolution efficiency and strategy efficiency. However, the learning algorithm of this research accumulates training errors.

The work [18] carries out the review of the latest achievements of evolutionary strategy algorithms. As a result of the authors' analysis, it is necessary to improve the basic algorithms of evolution strategies such as crossover, mutation and improve the algorithms of learning them.

The analysis of the works [9-18] showed that the vast majority of known studies are based on the use of general scientific approaches. Common limitations of these works are:

- failure to take into account the type of a priori uncertainty about the state of the analysis object;

- accumulation of learning errors (lack of learning algorithms);

- imperfection of standard procedures of the evolution strategy algorithm;

- high computational complexity;

- the inability to simultaneously solve the problem of finding the most appropriate structure and the most appropriate coefficients of the equation.

To create evaluation and identification software tools, it is necessary to create evaluation methods that must meet the following set of requirements:

- the possibility of forming a generalized evaluation indicator and choosing solutions based on sets of partial indicators, which change taking into account the complex multilevel evaluation structure;

- the possibility to aggregate heterogeneous evaluation indicators (both quantitative and qualitative) and choose solutions that differ in measuring scales and ranges of values;

- taking into account the compatibility and different significance of partial indicators in the generalized evaluation of decisions;

- taking into account the type of a priori uncertainty of the object state;

- flexible adjustment (adaptation) of evaluation models while adding (excluding) indicators and changing their parameters (compatibility and significance of indicators);

- no accumulation of evaluation errors due to the use of appropriate training methods.

All this leads to a combination of two approaches, namely:

- algorithm of evolution strategies - for structural and parametric assessment of the object state;

- evolving artificial neural networks - for training knowledge bases in order to increase the efficiency and reliability of the decision.

This will solve the problem of improving the efficiency and reliability of structural and parametric assessment of objects.

\section{The aim and objectives of the study}

The aim of the study is to develop a method of structural and parametric assessment of the object state, which would allow analyzing the object state. This will make it possible to increase the efficiency of assessing the object state and increasing the re-identification accuracy.
To achieve this aim, the following objectives were set:

- to conduct a formalized description of the structural and parametric assessment of the object state;

- to develop an algorithm for implementing the method of structural and parametric assessment of the object state;

- to conduct a practical test of the proposed method in the analysis of the operational situation of the troops (forces) grouping.

\section{Research materials and methods}

The research used the general provisions of the theory of artificial intelligence to solve the problem of analyzing the object state. Thus, the theory of artificial intelligence is the basis of the research.

The algorithm of evolution strategies was used to solve the problems of describing the state of dynamic objects. It describes how complex multi-level objects change over time. The research also uses the method of training artificial neural networks developed in previous works, which allows for deep training of artificial neural networks. The essence of deep learning is to train the architecture, type and parameters of the membership function. The simulation was performed using MathCad 2014 software (USA) and Intel Core i3 PC (USA).

\section{Results of research on the development of a method of} structural and parametric assessment of the object state

5. 1. Formalized description of structural and parametric assessment of the object state

Let the output of a dynamic object be represented by measurements forming a sample of the volume $s$, so $\left\{y_{i}, t_{i}\right\}$, $i=1, s$, where $y_{i} \in R, R$ is the measurement of the output of the dynamic system at time $t_{i} \in[0,+\infty), u=u(t)$ is the known control effect, which is the input of the dynamic system. The system is linear and is described by a linear differential equation as follows:

$$
a_{k} \cdot x^{(k)}+a_{k-1} \cdot x^{(k-1)}+\ldots+a_{0} \cdot x=b \cdot u(t), x(0)=x_{0} .
$$

It is necessary to determine the parameters of the system and the order $n$ of the differential equation, which is considered bounded, so $n-1 \leq M, M \in N$. It is assumed that in the system output measurement channel, there is a symmetrically distributed additive noise $\xi: M(\xi)=0, D(\xi)<\infty$, so $y_{i}=x\left(t_{i}\right)+\xi_{i}$.

Therefore, with an unknown order of the system, the problem of structural-parametric identification is solved, and the problem will be partially parameterized, because the maximum degree is included in the equation derivative determined in advance, limiting the dimension of the search space.

Assume that for a system of any order, its coefficient at the highest degree is equal to 1 , thus [19-23]:

$$
x^{(k)}+\frac{a_{k-1}}{a_{k}} \cdot x^{(k-1)}+\ldots+\frac{a_{0}}{a_{k}} \cdot x=\frac{b}{a_{k}} \cdot u(t) .
$$

Then, the solution of the structural-parametric identification problem will be found as a differential equation of order $m \leq M, M \in N$ as the solution of the Cauchy problem, under the given initial conditions [23, 24]: 


$$
\hat{x}^{(m)}+\hat{a}_{m} \cdot \hat{x}^{(m-1)}+\ldots+\hat{a}_{1} \cdot \hat{x}=\hat{a}_{0} \cdot u(t), \hat{x}(0)=x_{0},
$$

with the parameters $\hat{a}=\left(\begin{array}{lllll}0 & \ldots & \widehat{a}_{m} \ldots \hat{a}_{1} \hat{a}_{0}\end{array}\right)^{T} \in R^{n}$, so $n=M+1$, add the extremum of the selected function:

$$
\begin{aligned}
& I_{1}(a)=\sum_{i=1}^{N}\left|y_{i}-\hat{x}\left(t_{i}\right)\right|_{\bar{a}=a} \rightarrow \min _{a \in R^{n}} \\
& I_{2}(a)=\max _{i} \mid y_{i}-\hat{x}\left(t_{i}\right) \|_{\bar{a}=a} \rightarrow \min _{a \in R^{n}}
\end{aligned}
$$

To evaluate the process model, it is necessary to have information about the initial position of the system so that the Cauchy problem can be solved. In general, the vector of the initial position of the system, if it is not initially known, can be quantified, which, of course, is not always possible and largely depends on the properties of the sample.

Another way to determine the initial position of the system is to include the vector in the optimization problem. As a rule, for many problems, the observation process begins with the usual mode, therefore, all coordinates of the initial position for a dynamic system are equal to 0 , except for the position of the output system.

The control function is known for many systems, and in the case when the control is a process, the analytical form of which is unknown, it is necessary to approximate the function according to observations. The approach considered in the work can be generalized to the solution of the identification problem under unknown control, which will, accordingly, complicate the problem.

Such criteria, based on minimizing the discrepancies between sample data and system outputs, can be used to identify the elements of the matrices of the system of differential equations, which is selected as a process model with several dependent outputs [25, 26].

Let us construct a mathematical model of a dynamic process, which for convenience is presented in matrix form:

$$
\tilde{x}^{\prime}=\widehat{A} \cdot \tilde{x}(t)+\widehat{B} \cdot u(t), x(0)=x_{0},
$$

where $\hat{A}=\left(\widehat{a}_{i j}\right)_{i=1, j=1}^{n, n}$ is the matrix of a system of linear differential equations; $\widehat{B}=\left(\widehat{b}_{i j}\right)_{i=1, j=1}^{n, m}$ is the matrix of right parts, which are control factors; $\tilde{x}(t) \in R^{n}$ is the system state model; $u(t) \in R^{m}$ is the control actions represented as a vector function.

Given that there are several different system outputs, which may differ in the response amplitude, it is necessary to normalize each individual criterion. Then, the criterion has the following form:

$$
I(a)=\left.\sum_{j=1}^{N_{0}} \frac{\sum_{i=1}^{s_{j}}\left|y_{i}^{j}-\hat{x}^{j}\left(t_{i}^{j}\right)\right|}{\sup \left(|a-b|: a, b \in Y^{j} \bigcup x_{0}^{j}\right)}\right|_{\bar{A}=A, \bar{B}=B} \rightarrow \min _{A, B},
$$

$N_{0}$ is the number of outputs of the dynamic system; $s_{j}, \quad j=\overline{1, N_{0}}$ is the sample size for each output of the dynamic system; $y_{i}^{j}, i=\overline{1, s_{j}}, j=\overline{1, N_{0}}$ are the measurements of the outputs that form the samples; $t_{i}^{j}, i=\overline{1, s_{j}}$, $j=\overline{1, N_{0}}$ are the measurement times for each $j$-th output; $\sup \left(|a-b|: a, b \in Y^{j} \bigcup x_{0}^{j}\right)$ is the diameter of the set of measurement data for each output; $\left.\hat{x}^{j}(t)\right|_{\tilde{A}=A \tilde{B}=B}$ is the $j$-th output of the model for matrices $A, B$.

Criterion (6) is similar to the criterion for a single-input and single-output problem as (3). However, criterion (4) can also be generalized in the case of multiple input-output systems [27-30].

Thus, the problem of identifying a dynamic object was reduced to the problem of finding the extremum in the space of vectors with real coordinates. In this case, the peculiarity of representing the object structure leads to complex behavior of the objective function at the edges of some points in space, for which the first coordinates of the vector become 0 .

5. 2. Development of an algorithm for implementing the method of structural-parametric assessment of the object state

To create a method of structural-parametric assessment of the object state based on the formalization of structuralparametric assessment of the object state, an algorithm for implementing the method of structural-parametric assessment of the object state is proposed, shown in Fig. 1.

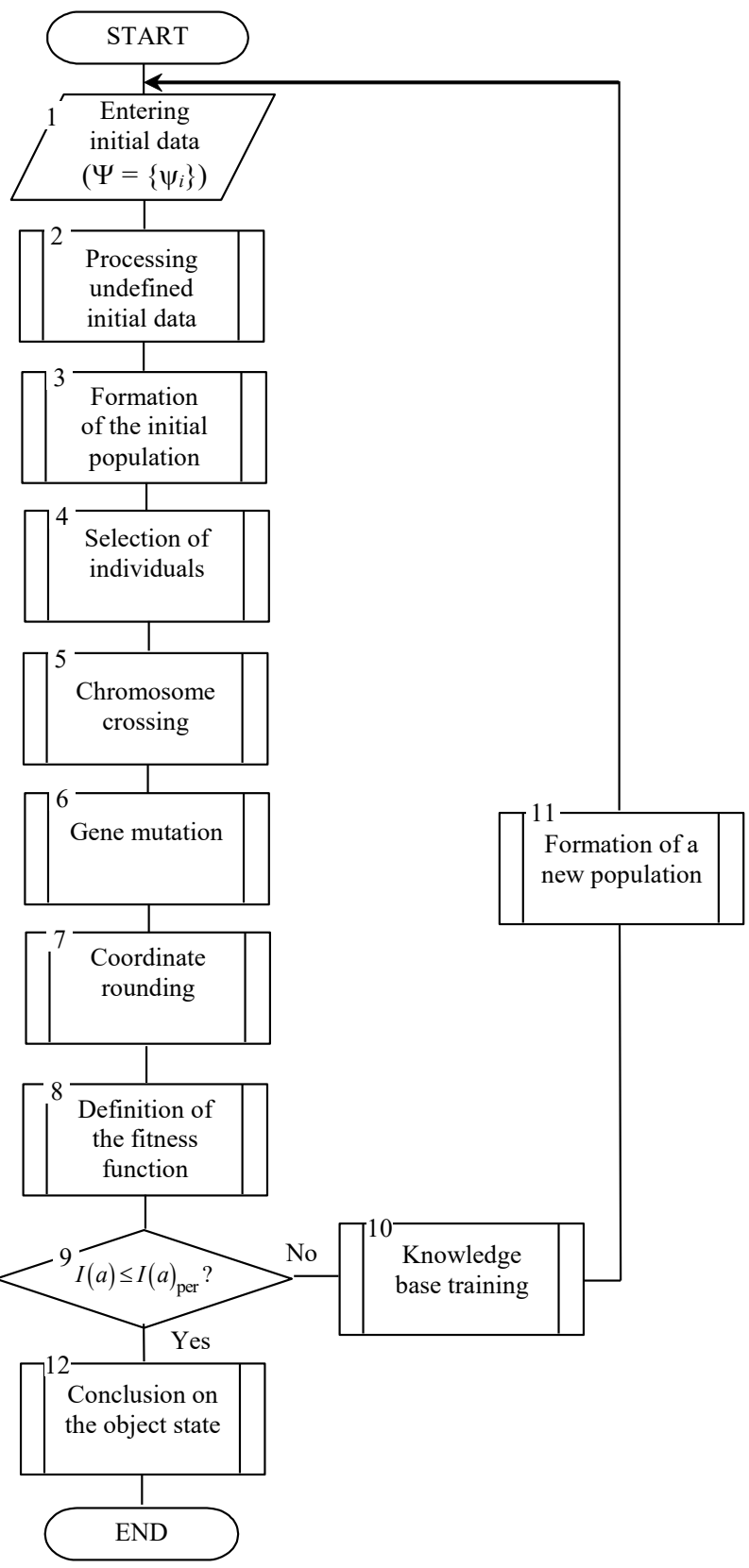

Fig. 1. Algorithm for implementing the object state analysis method 
1. Entering initial data (step 1 in Fig. 1). At this stage, initial available data about the object to be analyzed are entered. The basic object model is also initialized.

2. Processing initial data taking into account uncertainty (step 2 in Fig. 1).

At this stage, the type of uncertainty about the analysis object state is taken into account and the basic model of the object state is initialized [21,31]. The degree of a priori uncertainty can be: full awareness; partial uncertainty; complete uncertainty.

3. Formation of the initial population (step 3 in Fig. 1).

In this research, the procedure for generating the initial population is modified. Consider a modified procedure for generating the initial population.

Step 3. 1. For each individual, the order of the differential equation is chosen with probability $1 / M$.

Step 3.2. For the selected order $i_{\text {order }}$, each nonzero coordinate of the solution is played evenly on the interval $[-5,5]$.

Step 3. 3. All strategic parameters of the individual are played out evenly on the interval [0,1].

The proposed option to form the initial population allows forming solutions in the population corresponding to equations of one order. This, in turn, allows increasing the efficiency of decision-making by focusing the search according to the structure of the object described by the equation of the corresponding order.

4. Selection of individuals (step 4 in Fig. 1).

At this stage, several individuals are selected according to the selected selection scheme based on the fitness values of individuals in the current population.

At this stage, three types of selection are performed in parallel, namely: tournament, rank and proportional selection. In contrast to the standard algorithm of evolution strategies, in addition to proportional selection, two other types of individual selection are performed at this stage.

Parallel execution of three types of selection allows you to avoid two problems: random search - if the best individuals are neglected; search narrowing - while selecting only the best individuals from the population.

In tournament selection, $n_{t}$ different individuals are randomly selected from the current population, where $n_{t}$ is the tournament size, then the best individual in the selected group according to the fitness function is selected:

$$
\begin{aligned}
& H_{\text {parents }}=H_{i_{\text {toumament }}}, \\
& i_{\text {tournament }}=\max _{i \leq n_{t}}\left\{\begin{array}{l}
I_{n_{t}}^{i}, i=\overline{1, n_{t}}: P\left(I_{n_{t}}^{i}=1\right)= \\
=P\left(I_{n_{t}}^{i}=2\right)=\ldots=P\left(I_{n_{t}}^{i}=N\right)=1 / N
\end{array}\right\} .
\end{aligned}
$$

In proportional selection, each individual is assigned a probability to be selected in proportion to the value of its fitness function, so the probability of the $i$-th individual to be selected is found by the formula:

$$
\begin{aligned}
& p_{i}^{p}=\frac{\phi\left(f i t\left(o p_{i}\right)\right)}{\sum_{j=1}^{N} \phi\left(f i t\left(o p_{i}\right)\right)}, \\
& H_{\text {parents }}=H_{i_{\text {proportional }}}, \quad\left(i_{\text {proportional }}=j\right)=p_{j}^{p}, j=\overline{1, N} .
\end{aligned}
$$

The probability for each individual to be selected is determined by rank selection using the following formula:

$$
\begin{aligned}
& p_{i}^{r}={\frac{\phi(i)}{\sum_{j=1}^{N} \phi(j)}}^{\phi(x)=x}=\frac{2 \cdot i}{N(N+1)}, \\
& H_{\text {parents }}=H_{i_{\text {rank }}}, P\left(i_{\text {rank }}=j\right)=p_{j}^{r}, j=\overline{1, N} .
\end{aligned}
$$

For convenience, we assume that the genes of the offspring are determined by the tuple:

$$
H_{\text {crossover }}(i, j)=<o p_{\text {crossover }}^{i, j}, s p_{\text {crossover }}^{i, j}>,
$$

where $i, j$ are the parent indices in an ordered set of decisions; $o p_{\text {crossover }}^{i, j}$ is the objective parameters of the offspring; $s p_{\text {crossover }}^{i, j}$ is the strategic parameters of the offspring.

At the end of the selection procedures, the obtained decisions are compared according to the fitness criterion of the decision with the allowable efficiency of calculations.

5. Chromosome crossover (step 5 in Fig. 1).

At this stage, according to the chosen crossover scheme, the chromosomes of the selected parent individuals in some way form the offspring chromosome. At this stage, two crossover schemes are used, which are performed in parallel:

- crossover of the weighted sum of parameters;

- crossover of a random objective or strategic parameter of the offspring.

While performing only one crossover scheme, one of the best genotypes is determined, which in turn makes it impossible to make another decision. This is due to the fact that each individual will have the best genotype.

List various crossover schemes for $n_{p}$ parents. The first one is based on a weighted sum of parameters $j=\overline{1, q}$ :

$$
\begin{aligned}
& \left(o p_{\text {crossover }}\right)_{j}=\sum_{i=1}^{n_{p}} w_{i} \cdot\left(o p_{\text {parents }}\right)_{i} ; \\
& \left(s p_{\text {crossover }}\right)_{j}=\sum_{i=1}^{n_{p}} w_{i} \cdot\left(s p_{\text {parents }}\right)_{i} .
\end{aligned}
$$

The second scheme is based on the random selection of an objective or strategic parameter of the offspring from one of the parents, so the random variable has the following distribution probabilities: $P(i=k)=w_{k}, k=\overline{1, n_{p}}$ then for $k=\overline{1, n_{p}}, j=\overline{1, q}$ :

$$
\begin{aligned}
& P\left(o p_{\text {crossover }}\right)_{j}=\left(o p_{\text {parents }}\right)_{i} ; \\
& P\left(s p_{\text {crossover }}\right)_{j}=\left(s p_{\text {parents }}\right)_{i} .
\end{aligned}
$$

Thus, changing the method of parameter selection, we obtain different types of crossover, and for each type of crossover, it is necessary to meet the condition of normalization of parameters $\sum_{i=1}^{n_{p}} w_{i}=1$ then for $j=\overline{1, q}$ we obtain the following types of forming weighting factors:

- average:

$w_{j}=\frac{1}{n_{p}} ;$

- weighted average by fitness:

$$
\omega_{j}=\frac{f i t\left(\left(o p_{\text {parents }}\right)_{j}\right)}{\sum_{i=1}^{n_{p}} f i t\left(\left(o p_{\text {parents }}\right)_{i}\right)} ;
$$


- weighted average with random parameters and random by fitness, respectively:

$$
\begin{aligned}
& w_{j}=\frac{r_{j}}{\sum_{i=1}^{n_{p}} r_{i}}, r_{j} \sim U(0,1), \\
& w_{j}=\frac{r_{j}}{\sum_{i=1}^{n_{p}} r_{i}}, r_{i} \sim U\left(0, \text { fit }\left(\left(o p_{\text {parents }}\right)_{j}\right)\right) .
\end{aligned}
$$

\section{Gene mutation (step 6 in Fig. 1).}

At this stage, the genes of the offspring mutate. The difference of the proposed mutation order is that in this method:

- two mutation procedures, adaptive mutation and standard mutation, are performed in parallel;

- in each type of mutation, each pair of parameters: the objective and the corresponding strategic parameter mutate with a certain, predetermined probability, namely the probability of knowing a priori information about the object state.

Using the mutation operator, a new point of the search space is determined, which is the result of a random change in the coordinates of the generating point. For convenience of designation, the offspring:

$$
\tilde{H}=<o \tilde{p}, s \tilde{p}>=<o p_{\text {crossover }}, s p_{\text {crossover }}>,
$$

and the individual after mutation:

$$
H_{\text {mutation }}=<o p_{\text {mutation }}, s p_{\text {mutation }}>\text {. }
$$

The genes of the mutant individual are set by the following operations:

$$
\begin{aligned}
& o p_{\text {mutation }}=\left(o_{1}+N r_{p}^{1}, \ldots, o_{q}+N r_{p}^{q}\right), \\
& s p_{\text {mutation }}=\left(s_{1} \cdot e^{\tau \cdot N r_{s}^{1}}, \ldots, s_{q} \cdot e^{\tau \cdot N r_{s}^{q}}\right),
\end{aligned}
$$

where $N r_{p}^{i} \sim N\left(0, s_{i}^{2}\right), i=\overline{1, q}$ is the normally distributed onedimensional random variable, centered, standard deviation $s_{i}$; $N r_{s}^{i} \sim N(0,1), i=1, q$ is the normally distributed one-dimensional random variable, centered and with a single standard deviation; $\tau \in R$ is the adaptation parameter of the mutation operator. Unless otherwise specified, it is equal to 1.

For some complex extremum search tasks, another mutation scheme for strategic parameters will be more effective:

$$
s p_{\text {mutation }}=\left(\left|s_{1}+\tau \cdot N r_{s}^{1}\right|, \ldots,\left|s_{q}+\tau \cdot N r_{s}^{q}\right|\right) .
$$

An adaptive mutation is often used, where the adaptation parameter also undergoes equally probable random changes. For convenience, we denote the mutation parameter as $A \in R^{q}$, where $A$ is a random vector, and:

$$
P\left(A_{i}=\frac{A_{i}}{2}\right)=P\left(A_{i}+2 \cdot A_{i}\right)=0.5, i=\overline{1, q} .
$$

Then the mutation for the strategic parameters of the algorithm can occur as follows:

$$
\begin{aligned}
& s p_{\text {mutation }}=\left(A_{1} \cdot s_{1} \cdot e^{N r_{s}^{1}}, \ldots, A_{q} \cdot s_{q} \cdot e^{N r_{s}^{q}}\right), \\
& s p_{\text {mutation }}=\left(\left|s_{1}+A_{1} \cdot N r_{s}^{1}\right|, \ldots,\left|s_{q}+A_{q} \cdot N r_{s}^{q}\right|\right) .
\end{aligned}
$$

It is proposed to modify the mutation operator so that each pair of parameters: the objective and the corresponding strategic parameter mutate with a certain, predetermined probability, namely the probability of knowing a priori information about the object state. Let $z$ be a random vector, each coordinate of which takes one of two values:

$$
\forall i \leq q: P\left(z_{i}=0\right)=p_{m}, P\left(z_{i}=1\right) 1-p_{m},
$$

$p_{m}$ is the probability for each pair of genes to mutate.

Thus, the mutation will occur according to the following scheme (to simplify the representation, we use Hadamard multiplication):

$$
\begin{aligned}
& o p_{\text {mutation }}=o p_{\text {crossover }}+z \circ N r_{p}, \\
& s p_{\text {mutation }}=a b s\left(s p_{\text {crossover }}+z \circ\left(A \circ N r_{s}\right)\right), \\
& s p_{\text {mutation }}=a b s\left(s p_{\text {crossover }}+\tau \cdot z \circ N r_{s}\right),
\end{aligned}
$$

where $y=a b s(x): y_{i}=\left|x_{i}\right|, x_{i} \in R, i=\overline{1, q}$.

In the case of adaptive mutation:

$$
s p_{\text {mutation }}=a b s\left(s p_{\text {crossover }}+z \circ\left(A \circ N r_{s}\right)\right) \text {. }
$$

Similarly, for the standard mutation scheme:

$$
s p_{\text {mutation }}=\left(A_{1} \cdot s_{1} \cdot e^{\tau \cdot z_{1} \cdot N r_{s}^{1}}, \ldots, A_{q} \cdot s_{q} \cdot e^{\tau \cdot z_{q} \cdot N r_{s}^{q}}\right) .
$$

The mutation operator has been changed so that changing the value of the parameter controlling the probability of mutation, the mutation intensity can be controlled and reduced, if perturbations of objective parameters lead to a deterioration of the objective function.

7. Rounding of coordinates (step 7 in Fig. 1).

One of the special modifications of the method was the introduction of the operation of rounding the coordinates of vectors, which is carried out taking into account the degree of awareness $\eta$ of the analysis object state:

$$
o p_{j}^{i}=\operatorname{round}\left(o p_{j}^{i}\right) \times \eta, j=\overline{1, n}, i=\overline{1, N_{I}},
$$

where round ( $\cdot$ ): $R \rightarrow R$ is the function that rounds a number to its nearest integer; $\eta$ is the degree of uncertainty of a priori information about the object state.

Such an operator, which affects the objective parameters of the algorithm, solves the problem of reducing the coordinates of the vector to integers. The rounding operator is applied immediately after the mutation operator and after rounding there is a local improvement of the newly obtained population. For the same reason, the mutation operator was modified.

The rounding operator (26) of each coordinate causes the accuracy of the solution to be lost, due to the mantissa clipping. In other words, the alternative is roughened. In order to compensate for the loss of accuracy and increase the efficiency of the algorithm as a whole, the coordinate descent performs such a number of steps that at the selected step length, the rounded coefficient is refined so that the value that preceded the whole is returned.

Since several different schemes for finding solutions to an extreme problem are considered, it is necessary to research each of them separately to determine the most effective one. 
8. Determination of the fitness function (step 8 in Fig. 1).

At this stage, the fitness function for the newly obtained offspring is determined. It is necessary to indicate some features of the transition from the objective function to the fitness function. According to the definition of the fitness function:

$$
Q\left(a_{1}\right)<Q\left(a_{2}\right) \Rightarrow \operatorname{fit}\left(Q\left(a_{1}\right)\right)>\operatorname{fit}\left(Q\left(a_{2}\right)\right), a_{1}, a_{2} \in R^{q},
$$

where $\operatorname{fit}(\cdot): F_{R} \rightarrow R, F_{R} \subset R$ is the fitness function for the criterion $Q(a) \rightarrow \min , \quad a \in A ; \quad Q(\cdot): A \rightarrow R$ is the objective function, expressed by the functional that is defined, in general, throughout the search space $A$. $A$ is the alternative space, which in the considered problems is presented as Euclidean space of dimension $q$.

9. Checking the criterion is met (step 9 in Fig. 1).

This step checks whether the values of criterion (6) are met.

10. Knowledge base training (step 10 in Fig. 1).

The basis of the knowledge base in the research is evolving artificial neural networks (ANN). In this procedure, ANN are trained using the evolving ANN training method developed by the authors in [2]. This method differs from the known ones in that it allows training not only synaptic weights, but also the parameters of the membership function together with the ANN architecture. This feature allows increasing the efficiency of the proposed method. After that, the already trained ANN provides information for the formation of a new initial population.

11. Conclusion about the object state (step 12 in Fig. 1).

A conclusion about the object state is formed and the algorithm is stopped.

\section{3. Practical verification of the proposed method in} the analysis of the operational situation of the troops (forces) grouping

A method of structural and parametric assessment of the object state is proposed. Simulation of the structural-parametric assessment method was made in accordance with the algorithm in Fig. 1 and expressions (6)-(27). The simulation of the proposed evaluation method was carried out in the MathCad 14 (USA) software environment. The task of the simulation was to assess the elements of the operational situation of the troops (forces) grouping. The elements of the operational structure of the troops (forces) grouping are:

- the number of echelons placed in depth;

- width on the front;

- the number of control points of each of the elements of the operational structure;

- location of control points (mobile or stationary version).

It is necessary to determine the type of operation of the troops (forces) grouping and the organizational and staff structure, as well as the nature of the grouping's actions.

Initial data for the grouping assessment using the proposed method:

- the number of information sources on the state of the monitoring object is 3 (radio monitoring devices, earth remote sensing devices and unmanned aerial vehicles). To simplify the simulation, the same number of each device was taken - 4;

- the number of information features that determine the state of the monitoring object is 12 . These parameters include: affiliation, type of organizational-staff formation, priority, minimum width on the front, maximum width on the front. The number of personnel, the minimum depth on the flank, the maximum depth on the flank, the total number of personnel, the number of weapon samples, the number of types of weapon samples and the number of radio communication devices are also taken into account;

- options of organizational-staff formations - company, battalion, brigade.

Indicate which parameters for each type of operator were considered. The method was tested in different types of selection: proportional, rank, tournament (volume $2 \%$, volume $15 \%$, volume $25 \%$ ); recombination: average, weighted average, discrete, random; at different mutation probabilities $p_{m} \in\{2 / 11,5 / 11,1 / 5,1\}$ and adaptive and standard types. To determine the most effective combination of settings for each individual scheme considered, all other search parameters must be the same. The size of the population was chosen equal to 50 , the number of populations is 50 . These data are taken in accordance with the approximate number of command posts of the operational and tactical grouping of troops (forces).

Several different optimization algorithms for solving the extreme problem (6) were compared. Among them: algorithm of evolution strategies; hybrid algorithm of evolution strategies; proposed methodology; hybrid modified algorithm of evolution strategies. The number of calculations of the objective function for evolution strategies was chosen equal to the number of measurements of the objective function for other algorithms whose cycles used local improvement.

Fig. 2 is a graph showing the average fitness values of the best solutions found within each scheme with the best average settings. The proposed technique, which includes the proposed modification schemes, significantly outperforms all other algorithms.

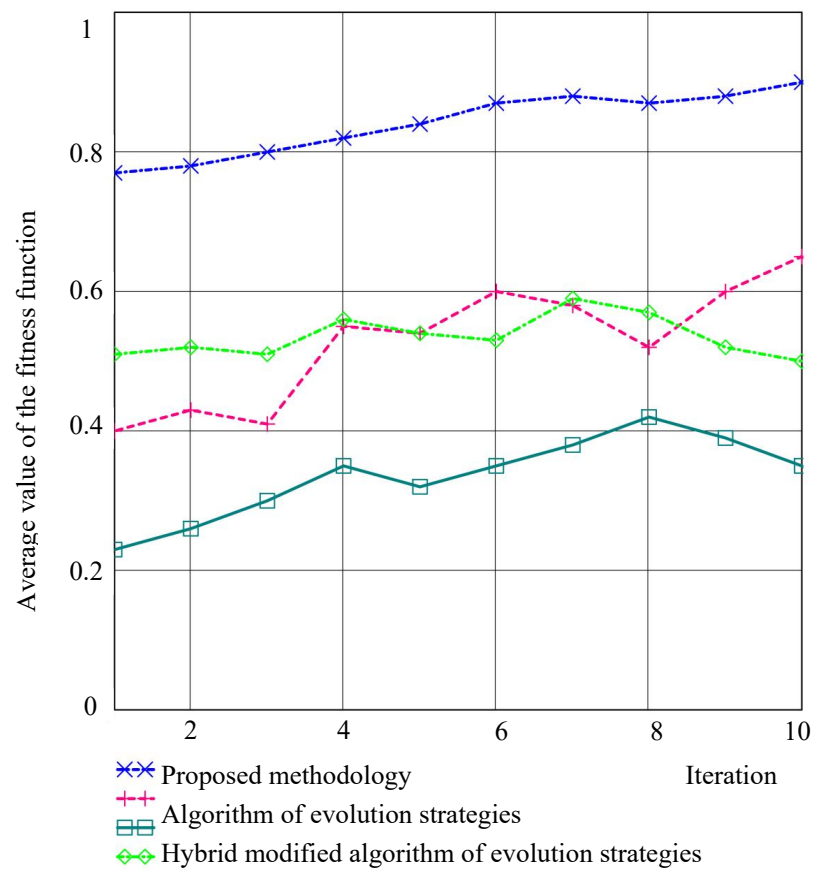

Fig. 2. Comparison graph of average fitness for different algorithms for solving the identification problem

As can be seen in Fig. 2, the average fitness of the solution increases as the order of the real object approaches the established limitation parameter of the maximum order of the model. It turns out that the algorithms should work in such a way as to preserve the ability to reduce the order of the system, maintaining the equality of the first coordinates to 0 . 
The fitness of the decision increases using the procedure for training artificial neural networks. The developed method is $30-35 \%$ more efficient in terms of fitness of the obtained solution compared to the conventional algorithm of evolution strategies. Also, the proposed method is $20-25 \%$ better than the modified algorithms of evolution strategies due to the use of additional improved procedures according to the fitness criterion of the obtained solution (Table 1).

Tables 2, 3 present comparative results of evaluating the training efficiency of evolving artificial neural networks.

Before training, the observation features were normalized at the interval $[0,1]$.
It is worth noting that the proposed training procedure showed a better PC (partition coefficient, PC) result compared to EFCM and a better operating time result compared to FCM. The research showed that this training procedure provides on average $10-18 \%$ higher training efficiency of artificial neural networks and does not accumulate errors during training (Tables 2, 3).

These results can be seen from the results in the last rows of Tables 2,3 as the difference in the Xie-Beni index. However, as it was already noted, the known methods accumulate errors, so the proposed method suggests the use of evolving artificial neural networks.

Table 1

Estimation of the probability of finding a solution that is close to the true one for different solution search algorithms

\begin{tabular}{|c|c|c|c|c|c|c|}
\hline \multirow{2}{*}{$\begin{array}{c}\text { Itera- } \\
\text { tion }\end{array}$} & $\begin{array}{c}\text { Proposed } \\
\text { method }\end{array}$ & $\begin{array}{c}\text { Algo- } \\
\text { rithm of } \\
\text { evolution } \\
\text { strategies }\end{array}$ & $\begin{array}{c}\text { Hybrid modi- } \\
\text { fied algorithm } \\
\text { of evolution } \\
\text { strategies }\end{array}$ & $\begin{array}{c}\text { Proposed } \\
\text { method }\end{array}$ & $\begin{array}{c}\text { Algo- } \\
\text { rithm of } \\
\text { evolution } \\
\text { strategies }\end{array}$ & $\begin{array}{c}\text { Hybrid modi- } \\
\text { fied algorithm } \\
\text { of evolution } \\
\text { strategies }\end{array}$ \\
\hline 1 & \multicolumn{4}{|c|}{$\hat{p}_{s}$} & \multicolumn{3}{|c|}{ Average fitness } \\
\hline 2 & 0.75 & 0.545 & 0.662 & 0.959344 & 0.66 & 0.79 \\
\hline 3 & 0.79 & 0.573 & 0.688 & 0.957950 & 0.678 & 0.8 \\
\hline 4 & 0.82 & 0.624 & 0.746 & 0.945785 & 0.713 & 0.83 \\
\hline 5 & 0.84 & 0.652 & 0.752 & 0.956173 & 0.728 & 0.839 \\
\hline 6 & 0.87 & 0.68 & 0.764 & 0.964235 & 0.735 & 0.843 \\
\hline 7 & 0.88 & 0.7 & 0.789 & 0.978653 & 0.746 & 0.858 \\
\hline 8 & 0.91 & 0.717 & 0.8032 & 0.983865 & 0.754 & 0.869 \\
\hline 9 & 0.93 & 0.744 & 0.814 & 0.992892 & 0.763 & 0.873 \\
\hline 10 & 0.96 & 0.768 & 0.83 & 1.000000 & 0.78 & 0.89 \\
\hline
\end{tabular}

Table 2

Comparative results of evaluating the training efficiency of artificial evolving neural networks

\begin{tabular}{|c|c|c|c|}
\hline System & $\begin{array}{c}\text { Algorithm } \\
\text { parameters }\end{array}$ & $\begin{array}{c}\text { XB (Xie-Beni } \\
\text { Index) }\end{array}$ & Time, $\mathrm{s}$ \\
\hline FCM (Fuzzy C-Means) & - & 0.1903 & 2.69 \\
\hline EFCM & Dthr $=0.24$ & 0.1136 & 0.14 \\
\hline EFCM & Dthr $=0.19$ & 0.1548 & 0.19 \\
\hline Proposed system (batch mode) & delta $=0.1$ & 0.0978 & 0.37 \\
\hline Proposed system (online mode) & delta $=0.1$ & 0.1127 & 0.25 \\
\hline
\end{tabular}

Comparative results of clustering

\begin{tabular}{|c|c|c|c|}
\hline System & $\begin{array}{c}\text { Algorithm } \\
\text { parameters }\end{array}$ & $\begin{array}{c}\text { XB (Xie-Beni } \\
\text { Index) }\end{array}$ & Time, $\mathrm{s}$ \\
\hline FCM (Fuzzy C-Means) & Dthr $=0.6$ & 0.2963 & 0.81 \\
\hline EFCM & Dthr $=0.6$ & 0.2330 & 0.54 \\
\hline Proposed system (batch mode) & delta $=0.4$ & 0.2078 & 0.45 \\
\hline Proposed system (online mode) & delta $=0.4$ & 0.2200 & 0.30 \\
\hline
\end{tabular}

6. Discussion of the results
of the development of
a method of structural
and parametric assessment
of the object state

A method of structural and parametric assessment of the object state is developed. This method allows assessing the structure and parameters of the object by solving the following partial research tasks:

- a formalized description of the structural-parametric assessment of the object state allows determining the relationship between the processes occurring during the structural-parametric assessment of the object state. The specified formalized description is given in expressions (1) $-(6)$;

- an algorithm for implementing the method of structural-parametric assessment of the object state is developed, which allows estimating (identifying) the structure and parameters of the object state. The main stages of implementing the methods are shown in Fig. 1 and expressions (7)-(27).

- a practical test of the proposed method in the analysis of the operational situation of the troops (forces) grouping is carried out. The results of practical testing of the proposed method showed an increase in the efficiency of structural and parametric assessment of the object state. Evaluation of the effectiveness of this method in the analysis of the operational situation of the troops (forces) grouping is shown in Fig. 2 and Tables 1-3.

Given the above, the problem of structural and parametric assessment of the object state with a given efficiency with an acceptable level of the decisions reliability can be considered solved. 
The main advantages of the proposed evaluation method are:

- a flexible hierarchical structure of indicators, which allows reducing the problem of multi-criteria evaluation of alternatives to one criterion or using a vector of indicators for selection;

- unambiguity of the obtained assessment of the object state, both the structure due to global assessment and parameters due to local assessment;

- wide scope of use (decision support systems);

- simplicity of mathematical calculations;

- no accumulation of training errors due to the evolution of the artificial neural network structure;

- the ability to adapt the system of indicators in the course of work;

- learning not only the synaptic weights of the artificial neural network, but also the type and parameters of the membership function;

- learning the architecture of artificial neural networks;

- calculation of data for one epoch without the need to store previous calculations;

- consideration of the type of uncertainty in constructing a fuzzy cognitive temporal model;

- the ability to synthesize the optimal structure of the decision support system.

The disadvantages of the proposed technique include:

- the maximum estimation accuracy is achieved after 8 estimation iterations;

- lower estimation accuracy based on a separate estimation parameter of the object state;

- lower estimation accuracy compared to other estimation methods.

This method allows:

- assessing the object state;

- identifying effective measures to improve management efficiency;

- increasing the speed of assessing the object state estimation;

- reducing the use of computing resources of decision support systems.

The developed method is 30-35\% more efficient in terms of the fitness of the obtained solution compared to with the classical algorithm of evolution strategies and by $20-25 \%$ than the modified algorithms of evolution strategies. According to the results of analyzing the effectiveness of the proposed method, its computational complexity is $12-18 \%$ less, compared to the methods for decision effectiveness estimation, presented in Table 2.

This study is a further development of research aimed at developing methodological principles for improving the efficiency of information and analytical support, published earlier [2, 4-6].

The limitations of this method include:

- availability of sufficient computing resources for calculations in accordance with the method procedures;

- availability of a knowledge base of the initial object state for the initial filling of knowledge bases and setting the coefficients of awareness about the object state.

\section{Conclusions}

1. A formalized description of the structural and parametric assessment of the object state was made. The problem of identifying a dynamic object was reduced to the problem of finding the extremum in the space of vectors with real coordinates. The peculiarity of representing the object structure leads to complex behavior of the objective function at the edges of some points in space, for which the first coordinates of the vector become 0 . This formalization allows describing the processes that take place in analyzing objects. The fitness of the obtained solution was chosen as a criterion for the effectiveness of this method.

2. The algorithm for implementing the method was defined, which allows:

- analyzing the object state under uncertainty;

- taking into account the initial type of uncertainty of initial data;

- conducting structural and parametric training of artificial neural networks for intelligent decision support systems.

The difference of the proposed algorithm is the use of advanced procedures for processing undefined initial data, selection, crossover, mutation, formation of the initial population, rounding of coordinates and procedures for learning the algorithm of evolution strategies.

3. We made a practical test of the proposed method in the analysis of the operational situation of the troops (forces) grouping on the example of assessing and forecasting the operational situation of the troops (forces) grouping. The developed method is $30-35 \%$ more efficient in terms of the fitness of the obtained solution compared to the conventional algorithm of evolution strategies. Also, the proposed method is $20-25 \%$ better than the modified algorithms of evolution strategies due to the use of additional improved procedures according to the fitness criterion of the obtained solution.

\section{Acknowledgments}

The author's team expresses gratitude for assistance in preparing the paper to:

- Doctor of Technical Sciences, Professor Kuvshinov Oleksiy - Deputy Head of the Educational and Scientific Institute of the Ivan Chernyakhovsky National Defense University of Ukraine;

- Doctor of Technical Sciences, Senior Researcher Sova Oleg - Head of the Department of Automated Control Systems of the Military Institute of Telecommunications and Informatization named after Heroes of Kruty;

- Doctor of Technical Sciences, Senior Researcher Zhuravskiy Yuriy - Head of the Department of Electrical Engineering and Electronics of the Zhytomyr Military Institute named after S. P. Korolyov;

- Honored Worker of Science and Technology of Ukraine, Doctor of Technical Sciences, Professor Slyusar Vadym - Chief Researcher of the Central Research Institute of Armaments and Military Equipment of the Armed Forces of Ukraine;

- Doctor of Technical Sciences, Professor Rothstein Oleksandr - Professor of the Jerusalem Polytechnic Institute named after Mahon Lev;

- PhD, Associate Professor Bashkyrov Oleksandr Leading Researcher of the Central Research Institute of Armaments and Military Equipment of the Armed Forces of Ukraine. 


\section{References}

1. Shyshatskyi, A. V., Bashkyrov, O. M., Kostyna, O. M. (2015). Development of integrated communication systems and data transfer for the needs of the Armed Forces. Weapons and military equipment, 1, 35-39.

2. Dudnyk, V., Sinenko, Y., Matsyk, M., Demchenko, Y., Zhyvotovskyi, R., Repilo, I. et. al. (2020). Development of a method for training artificial neural networks for intelligent decision support systems. Eastern-European Journal of Enterprise Technologies, 3 (2 (105)), 37-47. doi: https://doi.org/10.15587/1729-4061.2020.203301

3. Kuchuk, N., Mohammed, A. S., Shyshatskyi, A., Nalapko, O. (2019). The method of improving the efficiency of routes selection in networks of connection with the possibility of self-organization. International Journal of Advanced Trends in Computer Science and Engineering, 8 (1.2), 1-6. Available at: http://www.warse.org/IJATCSE/static/pdf/file/ijatcse01812sl2019.pdf

4. Pievtsov, H., Turinskyi, O., Zhyvotovskyi, R., Sova, O., Zvieriev, O., Lanetskii, B., Shyshatskyi, A. (2020). Development of an advanced method of finding solutions for neuro-fuzzy expert systems of analysis of the radioelectronic situation. EUREKA: Physics and Engineering, 4, 78-89. doi: https://doi.org/10.21303/2461-4262.2020.001353

5. Zuiev, P., Zhyvotovskyi, R., Zvieriev, O., Hatsenko, S., Kuprii, V., Nakonechnyi, O. et. al. (2020). Development of complex methodology of processing heterogeneous data in intelligent decision support systems. Eastern-European Journal of Enterprise Technologies, 4 (9 (106)), 14-23. doi: https://doi.org/10.15587/1729-4061.2020.208554

6. Shyshatskyi, A., Zvieriev, O., Salnikova, O., Demchenko, Y., Trotsko, O., Neroznak, Y. (2020). Complex Methods of Processing Different Data in Intellectual Systems for Decision Support System. International Journal of Advanced Trends in Computer Science and Engineering, 9 (4), 5583-5590. doi: https://doi.org/10.30534/ijatcse/2020/206942020

7. Sova, O., Shyshatskyi, A., Salnikova, O., Zhuk, O., Trotsko, O., Hrokholskyi, Y. (2021). Development of a method for assessment and forecasting of the radio electronic environment. EUREKA: Physics and Engineering, 4, 30-40. doi: https://doi.org/10.21303/ 2461-4262.2021.001940

8. Rodrigues, E., Gaspar, A. R., Gomes, Á. (2013). An evolutionary strategy enhanced with a local search technique for the space allocation problem in architecture, Part 2: Validation and performance tests. Computer-Aided Design, 45 (5), $898-910$. doi: https://doi.org/10.1016/j.cad.2013.01.003

9. Guerrero-Peña, E., Araújo, A. F. R. (2021). Dynamic multi-objective evolutionary algorithm with objective space prediction strategy. Applied Soft Computing, 107, 107258. doi: https://doi.org/10.1016/j.asoc.2021.107258

10. Pérez-González, C. J., Colebrook, M., Roda-García, J. L., Rosa-Remedios, C. B. (2019). Developing a data analytics platform to support decision making in emergency and security management. Expert Systems with Applications, 120, 167-184. doi: https://doi.org/10.1016/j.eswa.2018.11.023

11. Massel, L. V., Gerget, O. M., Massel, A. G., Mamedov, T. G. (2019). The Use of Machine Learning in Situational Management in Relation to the Tasks of the Power Industry. EPJ Web of Conferences, 217, 01010. doi: https://doi.org/10.1051/ epjconf/201921701010

12. Abaci, K., Yamacli, V. (2019). Hybrid Artificial Neural Network by Using Differential Search Algorithm for Solving Power Flow Problem. Advances in Electrical and Computer Engineering, 19 (4), 57-64. doi: https://doi.org/10.4316/aece.2019.04007

13. Osman, A. M. S. (2019). A novel big data analytics framework for smart cities. Future Generation Computer Systems, 91, 620-633. doi: https://doi.org/10.1016/j.future.2018.06.046

14. Mishchuk, O. S., Vitynskyi, P. B. (2018). Neural Network with Combined Approximation of the Surface of the Response. Research Bulletin of the National Technical University of Ukraine «Kyiv Politechnic Institute», 2, 18-24. doi: https://doi.org/ 10.20535/1810-0546.2018.2.129022

15. Kazemi, M., Faezirad, M. (2018). Efficiency estimation using nonlinear influences of time lags in DEA Using Artificial Neural Networks. Industrial Management Journal, 10 (1), 17-34. doi: https://doi.org/10.22059/imj.2018.129192.1006898

16. Prokoptsev, N. G., Alekseenko, A. E., Kholodov, Y. A. (2018). Traffic flow speed prediction on transportation graph with convolutional neural networks. Computer Research and Modeling, 10 (3), 359-367. doi: https://doi.org/10.20537/2076-7633-201810-3-359-367

17. Wu, M., Zhu, X., Ma, L., Wang, J., Bao, W., Li, W., Fan, Z. (2021). Torch: Strategy evolution in swarm robots using heterogeneous-homogeneous coevolution method. Journal of Industrial Information Integration, 100239. doi: https://doi.org/10.1016/ j.jii.2021.100239

18. Li, Z., Lin, X., Zhang, Q., Liu, H. (2020). Evolution strategies for continuous optimization: A survey of the state-of-the-art. Swarm and Evolutionary Computation, 56, 100694. doi: https://doi.org/10.1016/j.swevo.2020.100694

19. Rybak, V. A., Shokr, A. (2016). Analysis and comparison of existing decision support technology. System analysis and applied information science, 3, 12-18.

20. Poloziuk, K., Yaremenko, V. (2020). Neural networks and Monte-Carlo method usage in multi-agent systems for sudoku problem solving. Technology Audit and Production Reserves, 6 (2 (56)), 38-41. doi: https://doi.org/10.15587/2706-5448.2020.218427 
21. Akanova, A., Kaldarova, M. (2020). Impact of the compilation method on determining the accuracy of the error loss in neural network learning. Technology Audit and Production Reserves, 6 (2 (56)), 34-37. doi: https://doi.org/10.15587/2706-5448.2020.217613

22. Leoshchenko, S., Oliinyk, A., Subbotin, S., Zaiko, T. (2020). Usage of swarm intelligence strategies during projection of parallel neuroevolution methods for neuromodel synthesis. Technology Audit and Production Reserves, 5 (2 (55)), 12-17. doi: https://doi.org/ 10.15587/2706-5448.2020.214769

23. Yaremenko, V., Syrotiuk, O. (2020). Development of a multi-agent system for solving domain dictionary construction problem. Technology Audit and Production Reserves, 4 (2 (54)), 27-30. doi: https://doi.org/10.15587/2706-5448.2020.208400

24. Lakhno, V., Sagun, A., Khaidurov, V., Panasko, E. (2020). Development of an intelligent subsystem for operating system incidents forecasting. Technology Audit and Production Reserves, 2 (2 (52)), 35-39. doi: https://doi.org/10.15587/2706-5448.2020.202498

25. Hou, N., He, F., Zhou, Y., Chen, Y., Yan, X. (2018). A Parallel Genetic Algorithm With Dispersion Correction for HW/SW Partitioning on Multi-Core CPU and Many-Core GPU. IEEE Access, 6, 883-898. doi: https://doi.org/10.1109/access.2017.2776295

26. Nobile, M. S., Cazzaniga, P., Besozzi, D., Colombo, R., Mauri, G., Pasi, G. (2018). Fuzzy Self-Tuning PSO: A settings-free algorithm for global optimization. Swarm and Evolutionary Computation, 39, 70-85. doi: https://doi.org/10.1016/j.swevo.2017.09.001

27. Nugroho, E. D., Wibowo, M. E., Pulungan, R. (2017). Parallel implementation of genetic algorithm for searching optimal parameters of artificial neural networks. 2017 3rd International Conference on Science and Technology - Computer (ICST). doi: https://doi.org/10.1109/icstc.2017.8011867

28. Bergel, A. (2020). Neuroevolution. Agile Artificial Intelligence in Pharo, 283-294. doi: https://doi.org/10.1007/978-1-4842-5384-7_14

29. Lovska, A. (2015). Peculiarities of computer modeling of strength of body bearing construction of gondola car during transportation by ferry-bridge. Metallurgical and Mining Industry, 1, 49-54. Available at: https://www.metaljournal.com.ua/assets/Journal/ english-edition/MMI_2015_1/10\%20Lovska.pdf

30. Lovska, A., Fomin, O. (2020). A new fastener to ensure the reliability of a passenger car body on a train ferry. Acta Polytechnica, 60 (6), 478-485. doi: https://doi.org/10.14311/ap.2020.60.0478

31. Koshlan, A., Salnikova, O., Chekhovska, M., Zhyvotovskyi, R., Prokopenko, Y., Hurskyi, T. et. al. (2019). Development of an algorithm for complex processing of geospatial data in the special-purpose geoinformation system in conditions of diversity and uncertainty of data. Eastern-European Journal of Enterprise Technologies, 5 (9 (101)), 35-45. doi: https://doi.org/10.15587/ 1729-4061.2019.180197 